\title{
A Cascade-Based Emergency Model for Water Distribution Network
}

\author{
Qing Shuang, ${ }^{1}$ Yongbo Yuan, ${ }^{2}$ Mingyuan Zhang, ${ }^{2}$ and Yisheng Liu ${ }^{1}$ \\ ${ }^{1}$ Department of Construction Management, Beijing Jiaotong University, Beijing 100044, China \\ ${ }^{2}$ Department of Construction Management, Dalian University of Technology, Dalian, Liaoning 116024, China
}

Correspondence should be addressed to Qing Shuang; qings@bjtu.edu.cn

Received 24 November 2014; Revised 17 March 2015; Accepted 17 March 2015

Academic Editor: Carlo Cattani

Copyright (C) 2015 Qing Shuang et al. This is an open access article distributed under the Creative Commons Attribution License, which permits unrestricted use, distribution, and reproduction in any medium, provided the original work is properly cited.

\begin{abstract}
Water distribution network is important in the critical physical infrastructure systems. The paper studies the emergency resource strategies on water distribution network with the approach of complex network and cascading failures. The model of cascade-based emergency for water distribution network is built. The cascade-based model considers the network topology analysis and hydraulic analysis to provide a more realistic result. A load redistribution function with emergency recovery mechanisms is established. From the aspects of uniform distribution, node betweenness, and node pressure, six recovery strategies are given to reflect the network topology and the failure information, respectively. The recovery strategies are evaluated with the complex network indicators to describe the failure scale and failure velocity. The proposed method is applied by an illustrative example. The results showed that the recovery strategy considering the node pressure can enhance the network robustness effectively. Besides, this strategy can reduce the failure nodes and generate the least failure nodes per time.
\end{abstract}

\section{Introduction}

Infrastructure system is a significant and complex system that supports the normal implementation of urban functions. Water supply system is one of the indispensable elements in infrastructure system. By the methods in complex network, a large number of the systems in the nature and society can be depicted in the form of network diagram, such as internet [1], power grid [2], and scientific citation network [3]. Hence, urban water supply system can be construed as a complex network with various physical edges. The performance of water distribution network (WDN) can be studied by the complex network theory [4].

The robustness of WDN refers to the ability to avoid the loss of functions and the ability to tolerate errors and failures after the whole network damage or the components damage. The study on the robustness is one of the typical issues in complex network [5]. Cascading failure, as a step-by-step failure process [6], turns out to be a topic of recent interest on robustness and network security [7]. A large amount of loads exist in WDN. The failure of a certain component (such as breakdown or attack) may redistribute the network load, which may further lead to the result that certain components suffer a failure caused by the exceeding of their bearing capacity. The failures of these components may be likely to result in a secondary failure and a chain reaction. After that, a large number of components and even the whole network may collapse, which cause severe damage to the network.

WDN is a geography-related distributed system. In correlation with geographic space, WDN is highly sensitive to natural disasters (e.g., earthquake, hurricane) or human attack (e.g., terrorist attack) [8]. In WDN, the failures of the critical components arising from the destruction may lead to people and property loss and even impact the economic and social development. With the study on the cascading failures of WDN, Adachi and Ellingwood [9] constructed the service function assessment model of the urban water distribution system under the impact of earthquake, with power system taken as the back-up measure. Sitzenfrei et al. [10] established a cascading risk map with GIS, which involves both hazard and cascade vulnerability. The applied research showed that the neglect of cascading events undervalues the risk in WDN. Yazdani and Jeffrey [11] argued the fact that WDN was a spatial organization network. The structure and vulnerability 
of the network were studied by complex network. Four benchmark water networks were measured with the network paths, cycles, efficiency, and connectivity. Yazdani et al. [12] studied the structure of Kumasi's WDN and quantified its connectivity and redundancy. The relationship between these metrics and network robustness illustrated that the simple topological measure only depicted partial network structure without the ability to describe network property completely. The assessment on the robustness of WDN should be further considered with hydraulic attributes. Hawick [13] argued that, after a series of optimizations and designs, WDN developed into a highly complex network. Shuang et al. [14] established the node vulnerability analysis model of WDN based on cascading failures in combination with the network and hydraulic properties. It was proved that this model could identify the critical nodes of the system effectively under the cascading effect. Shuang et al. [15] simulated the reliability peak and persistent time of cascade propagation in WDN. The reliability assessment of WDN under cascading effect should introduce system uncertainties.

It is urgent for the government and society to cope with the disasters and bring down the losses resulting from disasters. It is proved that the approach to prevent and control the spread of disaster events is to explore the evolutionary mechanism of disaster and then bring forward an effective strategy. In the cascading failure model of WDN, if the load exceeds the bearing capacity, system components fail, which may further trigger the redistribution of network load and then the secondary failures. In actual life, however, there always exist emergency response mechanisms to reduce the loss of failures and restore the normal service function. Upon the failures of WDN, external emergency forces can intervene in the failing components to cope with emergencies and assist in their restoration.

The paper studies the emergency recovery strategies on WDN with the approach of complex network and cascading failures. The model of cascade-based emergency for WDN is built. The cascade-based model considers the network topology analysis and hydraulic analysis to provide a more realistic result. A load redistribution function with emergency recovery mechanisms is established. From the aspects of uniform distribution, node betweenness, and node pressure, six recovery strategies are given to reflect the network topology and the failure information, respectively. The recovery strategies are evaluated with the complex network indicators to describe the failure scale and failure velocity. The proposed method is applied by an illustrative example to analyze the impact on recovery with the six strategies.

\section{The Cascade-Based Emergency Model of WDN}

2.1. The Topology Structure. Prior to the simulated computation of WDN, there is a need to store the graphic information in a certain way and then establish the model for the WDN. Characterized by the network-like topological structure, WDN can be analyzed by the graph theory. Since the water in pipe flows along a certain direction, WDN is a directed graph [16]. Water reservoirs, consumers, and tanks can be abstracted as nodes. Pipes, pumps, and valves can be represented as edges. The neighboring nodes are connected by pipes. The incidence matrix $N$ is used to describe the relationship between the nodes and pipes in the network. The number of rows is equivalent to the nodes, and the number of columns is equivalent to the pipes, respectively. $N_{i j}$ in the matrix can be expressed as

$$
N_{i j}= \begin{cases}1, & \text { Node } i \text { is the initial point of pipe } j \\ -1, & \text { Node } i \text { is the terminal point of pipe } j \\ 0, & \text { Node } i \text { is the unconnected with pipe } j .\end{cases}
$$

\subsection{Parameters}

2.2.1. Load. Load is a significant physical parameter in the cascading failure model. The dynamic load changes lead to the cascading failures of the critical infrastructures such as power grid, water distribution system, gas supply network, traffic network, and communication network. Load also exists on the components such as nodes and pipes in WDN. Both the excessively large and small loads result in the flow changes, which may further trigger a series of cascading failures. After attack, the WDN redistributes the water pressure and flow in the network according to the topological structure and hydraulic changes. The complex network distributes the network load according to betweenness. To further study the cascade propagation of WDN, the node service pressure $P^{\text {ser }}$ in the normal operation is used as the initial load [14].

2.2.2. Capacity. Capacity is the load that a node can bear. If the load exceeds its bearing capacity, components suffer from failures. These failures cause the flow redistribution, leading to secondary failures. With the increase in operation time and the expansion in urban size, WDN has been further expanded based on the original construction size. Meanwhile, water demand changes randomly with the requirements of urban population and industry. As a result of these, the node pressures are no longer the initial value but they will be left in a changing state. Therefore, for the water distribution system that keeps running for a long time, there is a need to take account of the changes in node pressure and measure the robustness of WDN by its new constraints. The node minimum capacity is defined as the acceptable minimum water pressure $P_{k}^{\min }$. The node maximum capacity is defined as follows:

$$
P_{k}^{\max }=(1+\alpha) P_{k}^{\mathrm{ser}},
$$

where $P_{k}^{\text {ser }}$ is the service pressure of node $k$. The service pressure can be obtained in normal operation. It ensures that all the water demand can be satisfied. The node maximum capacity refers to the upper limit of the nodes constrained by cost or aging. In (2), $\alpha \geq 0$ expresses the node tolerance, which implements the control over the intensity of initial load and load distribution. $\alpha$ has evaluated the extra pressure that a nodes in WDN can bear. $\alpha$ has presented a way to assess the performance of WDN from system perspective. The greater the value of $\alpha$ is, the huger the difference between 
the components will be; that is, the load distribution will be more nonuniform.

The research focuses on the dynamic changes in WDN. The network remains stable in the initial stage. The load of every node is smaller than its capacity. After the failure occurs, the load on the failure node is distributed to its neighboring nodes. If the neighboring nodes are unable to process the extra load (i.e., the load exceeds its capacity), the neighboring nodes fail and then result in cascading failures. Equation (3) describes the failure propagation process. If the redistributed node pressure is within its capacity, the node can still supply water; otherwise, the node is recognized as a new avalanched node. The connected pipes are closed to avoid risk expansion. Consider

$$
\text { New avalanched node }= \begin{cases}0, & P_{k}^{\min }<P_{k}<P_{k}^{\max } \\ 1, & \text { otherwise. }\end{cases}
$$

2.2.3. Actual Demand. The pressure-driven simulation method is based on the laws of conservation of mass and energy to determine pressure and flow distribution. The pressure-driven simulation method is effective in describing WDN which can prevent the negative water pressure under failure conditions. With the help of pressure-driven strategy, Wagner's model suggests that there is a relationship between actual flow and node pressure [17]. This function is widely applied with the high ability in calculating node demands $[18,19]$.

In Wagner's model, the demand supplied equals the required one as a customer controls a faucet when the system capacity is not exceeded [20]. The demand becomes the maximum allowed by the actual pressure in pressuredeficient condition. In addition, the node pressure should be neither too low nor too high. Abnormally high pressures may cause aging pipes to burst and lose service functions. Therefore, each node pressure must be controlled between the maximum pressure and the minimum pressure. When $P_{k}^{\max } \leq P_{k}$, the abnormally high-pressure condition occurs. To avoid risk expansion, it is assumed that the section where the failure demand node covered is isolated from the rest of the network. The failure node is removed out of the network and its connected pipes are closed. Hence, based on Wagner's model, the relationship between actual demand and node pressure is revealed in combination with the minimum and maximum pressures. Consider

$$
Q_{k, t}^{\text {act }}= \begin{cases}0 & P_{k, t} \leq P_{k}^{\mathrm{min}}, \\ Q_{k, t}^{\mathrm{req}} \sqrt{\frac{P_{k, t}-P_{k}^{\mathrm{min}}}{P_{k}^{\mathrm{ser}}-P_{k}^{\mathrm{min}}}} & P_{k}^{\mathrm{min}}<P_{k, t}<P_{k}^{\mathrm{ser}}, \\ Q_{k, t}^{\mathrm{req}} & P_{k}^{\mathrm{ser}} \leq P_{k, t}<P_{k}^{\max }, \\ 0 & P_{k}^{\mathrm{max}} \leq P_{k, t},\end{cases}
$$

where $Q_{k, t}^{\text {act }}$ is the actual demand of node $k$ at time $t . Q_{k, t}^{\text {req }}$ is the required demand of node $k$ at time $t$. The required demand is the full demand at the node. It can be obtained as the WDN performing normally. $P_{k, t}$ is the pressure of node $k$ calculated at time $t . P_{k}^{\min }$ is the minimum pressure of node $k . P_{k}^{\text {ser }}$ is the service pressure of node $k . P_{k}^{\max }$ is the maximum pressure of node $k$.

There is still a lack of mature and universal hydraulic analysis software that can support the pressure-driven simulation method. In consideration of this, the robustness of WDN under the condition of cascading failures is figured out in combination with EPANET 2 and the actual demand function.

\subsection{The Cascading Failure Model}

2.3.1. EPANET 2. EPANET 2 is used to run the hydraulic simulation. EPANET 2 [21] is open source software developed by the United States Environmental Protection Agency (EPA). EPANET 2 can simulate the water hydraulic and water quality of WDN in a certain period of time. The gradient algorithm proposed by Todini and Pilati [22] is combined in EPANET 2 to find a solution for steady flow equations. EPANET 2 has been widely applied because of its high computing speed and satisfactory simulation effect. In terms of academic study, EPANET 2 is nearly deemed as the standard computing engine due to the accurate computing result and good compatibility $[19,23,24]$.

The numerical simulation of WDN is implemented by calling Toolkit from EPANET 2 through MATLAB under the failure condition. EPANET 2 has provided the users with Toolkits, which is implemented by the way of dynamic link library (DLL) technology. It allows developers to customize computing engine of EPANET 2 according to their specific demands. The ".inp" output file of EPANET 2 has presented a targeted development approach that can be embedded in the optimal design or parameter analysis to increase the function of aided analysis. The process of MATLAB calling EPANET 2 Toolkits with pressure-driven strategy is shown in Figure 1.

2.3.2. Attack Pattern. Cascading failures can be triggered by random attack and intentional attack $[25,26]$. Random attack means destroying a network component randomly. Intentional attack means damaging a certain network component according to some selection strategies. The target of random attack is a network component which is selected randomly. The target of international attack is a network component with some special feature, for example, the most connected node, the highest betweenness node, or the lowest betweenness node. Attack on the special component can cause a more rapid network failure. These special components can be regard as vulnerability components. When the network information is entirely unknown, the components in the network can only be attacked randomly. However, in actual network, the vulnerability differs from component to component. When the network information can be completely or partially obtained, the network can be break down rapidly if priority is given to the vulnerable ones.

From the perspective of infrastructure protection, the intentional attack is chosen. The actual network shows high tolerance for random failures. As revealed by the researches of network security, the attack against a critical component is likely to cause cascading failures on large scale and even 


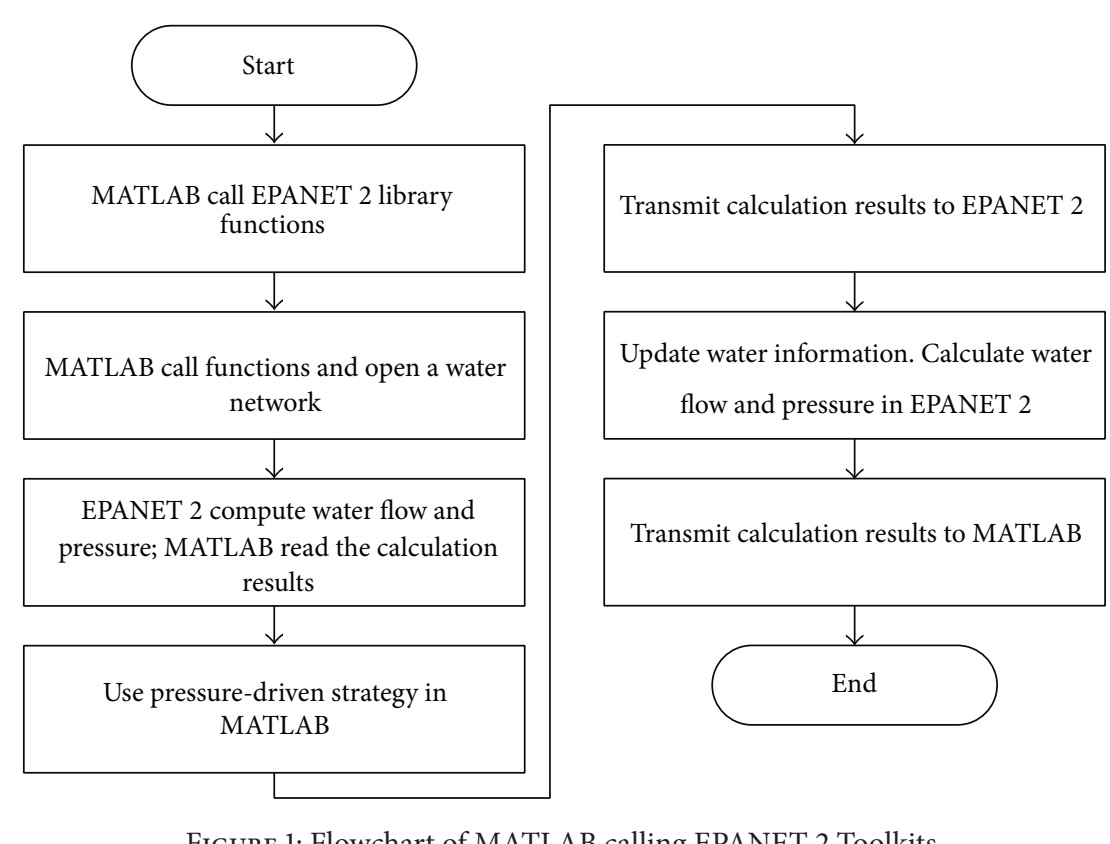

FIgURE 1: Flowchart of MATLAB calling EPANET 2 Toolkits.

the entirely network [6]. Both the random attack and the intentional attack can incur the loss of network performance. However, if the intentional attack happens to the critical components of the network, the destruction of the network performance is likely to occur.

Hence, the intentional attack is the choice in this paper. The attack target is constituted by the node-based attacks. The failure of the nodes or pipes in WDN causes changes to the topological structure and water properties. It is necessary to update the WDN model before the hydraulic analysis. The update of WDN involves two aspects: (1) the update of the topological structure, such as shutting down the pipes within the influencing scope of accidents and (2) recalculating the water demand within the influencing scope. Therefore, the influence of node failure in WDN can be described as follows: after node failure, the node will be isolated to prevent loss and spread; that is, the failure node is removed out of WDN. In EPANET 2, the actual demand is set as zero and the upstream and downstream pipes associated with the failure node are shut down. The stop of cascading failures of the WDN is defined as no new failure nodes or pipes appeared, that is, the network returns to stable state again. The flowchart of nodebased attack in WDN can be seen in Figure 2.

\subsection{The Emergency Model}

2.4.1. The Resource Threshold Value. New failure nodes appear after the intentional attack. The failure nodes may trigger the hydraulic redistribution of WDN. However, in real life, emergency response can help people to reduce losses. After a natural disaster, manpower with technical support, vehicles, and emergency supplies are involved in the recovery activities to fight the disaster. Manpower, vehicles, and emergency supplies can be regarded as external emergency resources. These external emergency resources play a signification role in network recovery and disaster relief.

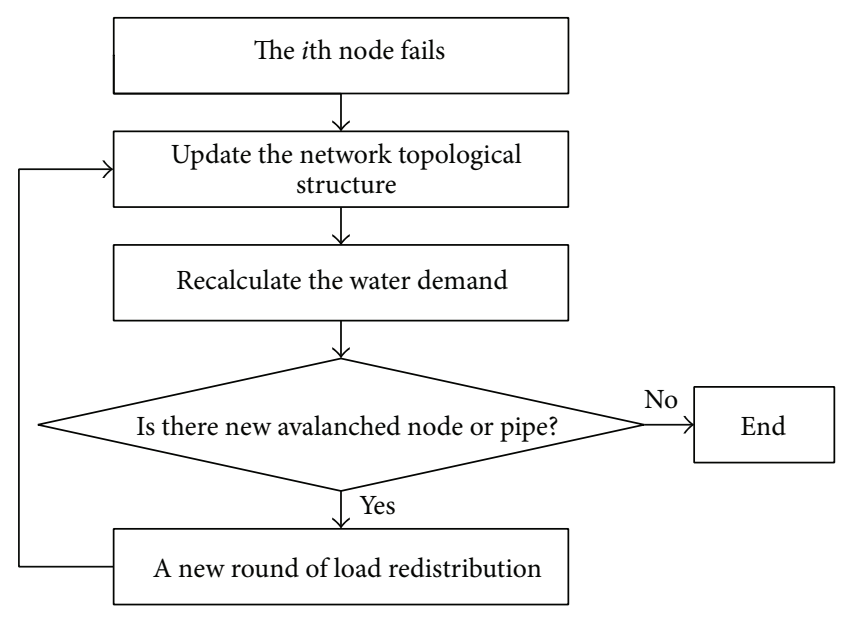

FIGURE 2: Flowchart of the node-based attack in WDN.

The threshold value of the emergence resources is defined. The threshold value of the low-pressure resources $P_{\min , k}^{\text {res }}$ is expressed as

$$
P_{\min , k}^{\mathrm{res}}=P_{k}^{\min } \times(1+\beta) .
$$

The threshold value of the high-pressure resources $P_{\max , k}^{\text {res }}$ is expressed as

$$
P_{\max , k}^{\mathrm{res}}=P_{k}^{\max } \times(1-\beta)
$$

where $P_{k}^{\min }$ is the minimum pressure of node $k, P_{k}^{\max }$ is the maximum pressure of node $k$, and $\beta$ is the parameter of external emergency resources. With the definition of the lowpressure resource threshold value and high-pressure resource threshold value, the original water pressure in WDN is expanded from three (i.e., minimum pressure, maximum 


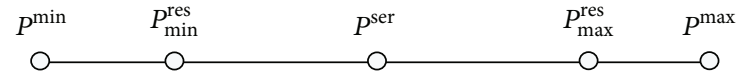

Figure 3: The relationship of node pressure.

pressure, and service pressure) to five. The relationship is shown in Figure 3.

(1) When the node pressure is smaller than the minimum pressure, the excessively low water pressure leads to flow interruption and short supply. The node actual demand becomes zero. External emergency resources can be introduced for restoration.

(2) When the node water pressure is greater than the minimum pressure but smaller than the low-pressure resource threshold, the node suffers low-pressure challenge. If the external resources are not introduced, the water pressure will keep declining and then lead to flow interruption. In this situation, external emergency resources can be introduced to restore the nodes within the interval of the water pressures mentioned above.

(3) When the node water pressure is higher than the lowpressure resource threshold but lower than the service pressure, the water is supplied in a reduction level. The actual demand can be calculated with (4).

(4) When the node water pressure is higher than the service water pressure but lower than the highpressure resource threshold, the node maintains normal supply. The actual demand is equal to the required demand.

(5) When the node water pressure is higher than the high-pressure resource threshold but lower than the maximum pressure, the node suffers high-pressure challenge. If the external resources are not introduced, the pipe burst may arise from the continuous increase in water pressure. In this situation, external emergency resources can be introduced to restore the nodes within the interval of the water pressures mentioned above.

(6) When the node water pressure is higher than the maximum pressure, it easily results in pipe burst and then cascading failures. Under this circumstance, the node fails and the actual flow is zero. In view of this, the node can be restored by the external emergency resources.

2.4.2. The Load Redistribution. The water pressures of every node can be obtained by the recalculation of network hydraulic under failure conditions. Judge the interval of water pressure where the node pressure exists. Suppose the water pressure of node $k$ is $P_{k, t}$ at time $t$. The external emergency resources should be distributed to the nodes with the recovery strategies if $P_{k, t}$ is greater than the maximum resource threshold or smaller than the minimum resource threshold. After restoration, the final load is $P_{k, t}^{\prime}$. The load redistribution process can be expressed as follows:

$$
P_{k, t}^{\prime}= \begin{cases}P_{k, t} \times\left(1+w_{k, t} \times R\right) & P_{k, t} \leq P_{k, \text { min }}^{\mathrm{res}}, \\ P_{k, t} & P_{k, \text { min }}^{\mathrm{res}}<P_{k, t}<P_{k, \text { max }}^{\mathrm{res}}, \\ P_{k, t} \times\left(1-w_{k, t} \times R\right) & P_{k, t} \geq P_{k, \text { max }}^{\mathrm{res}},\end{cases}
$$

where $w_{k, t}$ represents the weight for node $k$ at time $t$. The weight describes the resources that are introduced according to different recovery strategies. $R$ is the total available resources. According to (7), after the node is allocated with a certain quantity of emergency resources, the final load $P_{k, t}^{\prime}$ suggests that node $k$ only has three states, that is, the normal state $\left(P_{k \text {,min }}^{\text {res }}<P_{k, t}<P_{k, \text { max }}^{\text {res }}\right)$, the challenge state $\left(P_{k \text {,min }}<\right.$ $P_{k, t} \leq P_{k \text {,min }}^{\text {res }}$ or $\left.P_{k \text {,max }}^{\text {res }}<P_{k, t} \leq P_{k \text {,max }}\right)$, and the avalanched state $\left(P_{k, t} \leq P_{k, \text { min }}\right.$ or $\left.P_{k, t} \geq P_{k, \text { max }}\right)$.

The node with the challenge state can still ensure water supply. However, if the node suffers avalanche, the node fails and then it is removed out of the WDN.

2.4.3. The Recovery Strategies. The weight definition based on node betweenness in scale-free network can diminish the avalanched size resulting from cascading failures [27]. Hence, in this paper, weight is devoted to formulating the recovery strategies from three aspects including uniform distribution, node betweenness, and node pressure. The recovery strategies are considered from two aspects, that is, the network topological structure and the collapse information. The node betweenness is defined as follows:

$$
B_{k}=\sum_{a \neq b} \frac{\sigma_{a b}(k)}{\sigma_{a b}},
$$

where $\sigma_{a b}(k)$ is the shortest path between node $a$ and node $b$ passing through node $k . \sigma_{a b}$ is the sum of all the shortest paths between node $a$ and node $b$. In most cascading failure studies [28-30], node betweenness is used to measure the network topological property. It evaluates the node influence from the aspect of topology. The larger the node betweenness is, the greater the influence will be.

The recovery strategies (RSs) are described as follows.

RS 1: the resources are distributed to all nodes uniformly.

RS 2: the resources are distributed to all nodes according to the node betweenness.

RS 3: the resources are uniformly distributed to the challenged nodes.

RS 4: the resources are distributed to the avalanched nodes uniformly. If there are no avalanched nodes, the resources are uniformly distributed to the challenged nodes.

RS 5: the resources are distributed to the avalanched nodes according to node betweenness. If there are no avalanched nodes, the resources are distributed to the challenged nodes according to node betweenness. 
RS 6: the resources are distributed to the avalanched nodes according to the node pressure. If there are no avalanched nodes, the resources are distributed to the challenged nodes according to the node pressure.

RS 7: no emergency resource is introduced to the WDN.

Among all the strategies above, RS 2 is based on the network topological structure while RSs $3 \sim 6$ are based on the network topological structure and the breakdown information.

2.5. The Robustness Evaluation Index. The robustness indexes are introduced from [31].

The relative size of the nodes $G$ in the largest connected component is as follows:

$$
G=\frac{N^{\prime}}{N} .
$$

The avalanched size of nodes AS is as follows:

$$
\mathrm{AS}=\frac{\sum \mathrm{as}_{i}}{N} .
$$

The challenged size of nodes CS is as follows:

$$
\mathrm{CS}=\frac{\sum \mathrm{Cs}_{i}}{N} .
$$

The cascade propagation velocity is as follows:

$$
V=\frac{N-N^{\prime}}{T},
$$

where $N^{\prime}$ is the nodes in the largest connected component of WDN after the cascading failures stop, $N$ is the number of the demand nodes in WDN, $\sum \mathrm{as}_{i}$ and $\sum \mathrm{cs}_{i}$ are the number of the avalanched and the challenged nodes in WDN after the stop of the cascading failures resulting from the attack against node $i$, and $T$ is the total number of iterations that measure the time steps of cascade propagation in the network.

$G$ reflects the largest connected component after cascading failures. It can quantify the robustness of structure against cascading failures. The network has good robustness as $G$ approaches one. On the contrary, AS evaluates the avalanched size. The network has poor robustness as AS approaches one. CS describes the challenged condition. If effective recovery strategy can be given to these nodes, the possibility of network back to normal operation can be improved. In addition, $V$ measures the quantity of avalanched nodes in unit time. The smaller the $V$ is, the less the collapse nodes per unit time will be.

2.6. Simulation. The simulation process is described in Figure 4.

Step 1. Call the EPANET 2 Toolkits through MATLAB. Upload topological structure and basic data of WDN.
Step 2. Calculate the capacity according to (2) and the resource threshold value according to ((5)-(6)).

Step 3. Choose the initial failure node according to the attack strategy.

Step 4. Compute the water pressure of each node by EPANET 2.

Step 5. Record the new avalanched and challenged nodes resulting from the initial attack.

Step 6. Calculate the recovery amount of each node according to the recovery strategies.

Step 7. Introduce the emergency resources and restore node pressure according to (7).

Step 8. Update the actual demand according to the restored water pressure. The updated actual demands are used as node demand of next iteration.

Step 9. If there is new avalanched node or pipe, the iterations should continue. Otherwise, the cascading effect stops and attention should be switched to Step 10 .

Step 10. Repeat Steps 3-9 until all nodes failure have been simulated.

Step 11. Compute the robustness assessment indexes of WDN.

\section{Case Study}

A WDN from Shuang et al. [14] is used as an example. The network is represented as 17 nodes and 21 pipes. The network topological structure, node elevation, base demand, pipe diameter, length, and Hazen-William roughness are shown in Figure 5. The total head is $30 \mathrm{~m}$. The total pipe length is $23.55 \mathrm{~km}$. The pipe lengths range from $800 \mathrm{~m}$ to $1700 \mathrm{~m}$. The pipe diameters vary from $150 \mathrm{~mm}$ to $350 \mathrm{~mm}$. The base demands vary from $6.94 \mathrm{~L} / \mathrm{s}$ to $13.89 \mathrm{~L} / \mathrm{s}$. The minimum pressure of every node is $6 \mathrm{~m}$.

EPANET 2 is used to calculate the node pressure under normal condition. The node pressure is shown in Table 1 . The pressure of node 14 is the smallest; $P_{14}=7.12 \mathrm{~m}$. The pressure of node 1 is the largest; $P_{1}=30 \mathrm{~m}$.

The acceptable minimum pressure of all consumer nodes is $6 \mathrm{~m}$. The maximum pressure is figured out through (2). Since the original literature does not contain the maximum capacity, the value range of tolerance parameter $\alpha$ is defined as $0 \sim 1.0$ to cover all possible maximum capacities.

As shown in Figure 5, this WDN belongs to the smallsize network. Therefore, in order to evaluate the recovery strategies, the cascading failure process from node 1 to node 17 is simulated successively to calculate the relative size $G$, the avalanched size AS, the challenged size CS, and the cascade propagation velocity $V$ of every node until the network becomes stable again. The evaluation result with each $\alpha$ is the average [32] of evaluation index from node 1 to node 17. 


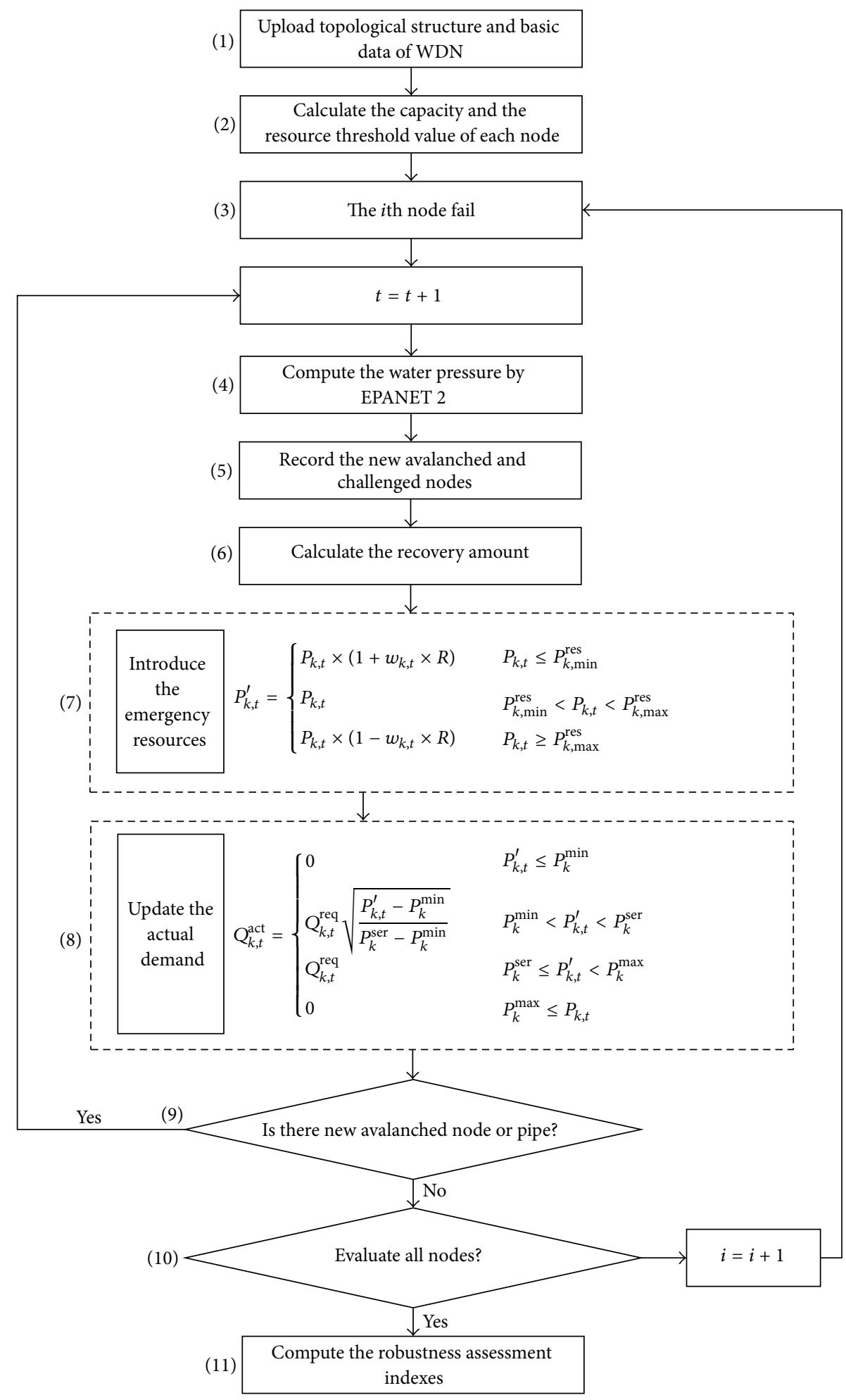

FIgURE 4: The flowchart of the cascade-based emergency model.

Set the total available resources as $R=0.5$. The simulation is implemented according to the flowchart shown in Figure 4. The simulation results can be seen in Figures 6-9.

Firstly, the black line in Figures 6-9 shows the network performance of WDN under cascading effect without emergency resource. It can be seen that despite the increase in tolerance parameter $\alpha$, the relative size $G$ without emergency resource is smaller than that at the time when the emergency resources are introduced. The avalanched size AS is higher than the emergency introduced situation. The challenged size 


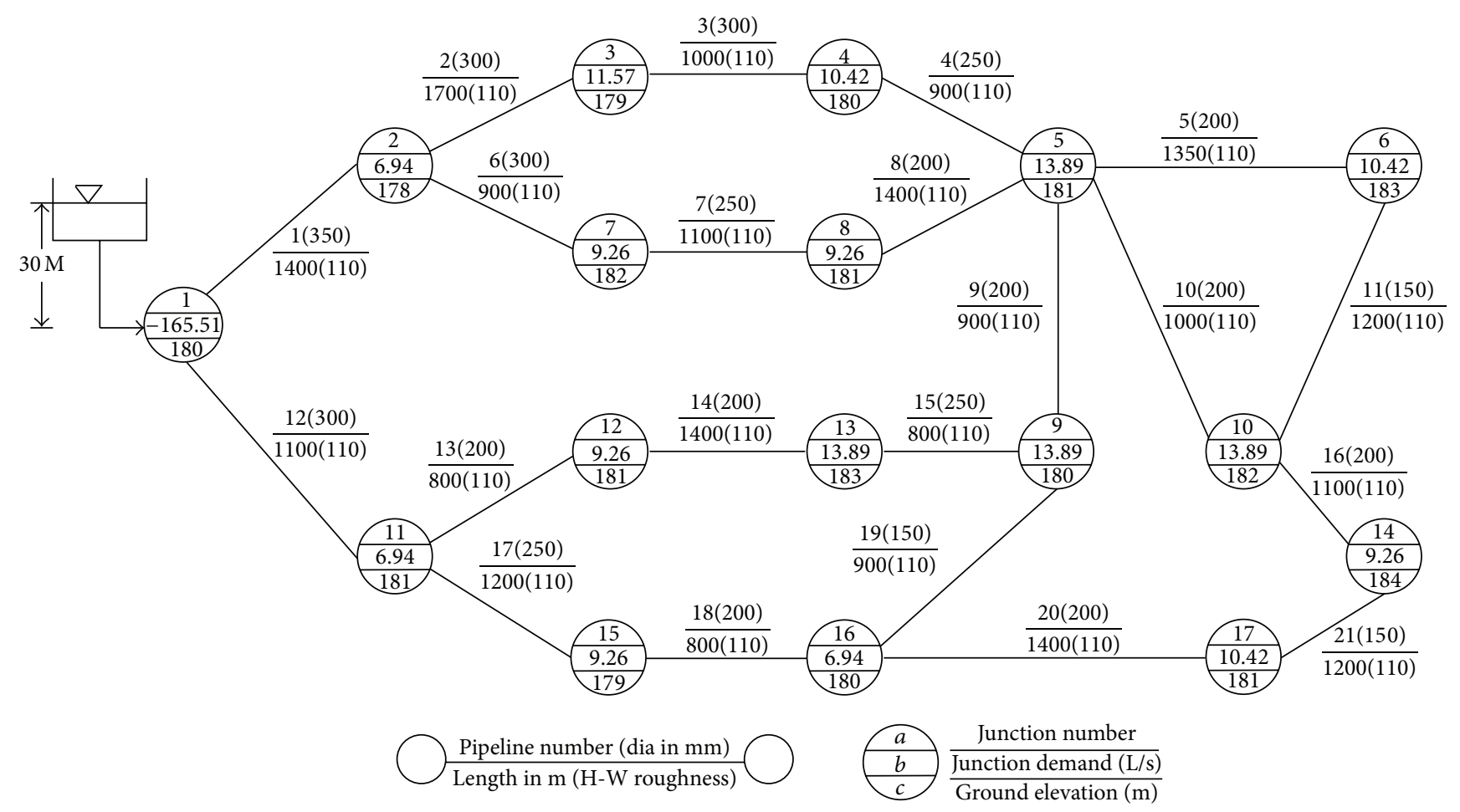

FIGURE 5: The layout of WDN.

TABLE 1: The node pressure under normal condition.

\begin{tabular}{lc}
\hline Node & Pressure $(\mathrm{m})$ \\
\hline 1 & 30.00 \\
2 & 25.58 \\
3 & 19.43 \\
4 & 16.55 \\
5 & 13.20 \\
6 & 8.89 \\
7 & 20.02 \\
8 & 18.26 \\
9 & 13.91 \\
10 & 9.48 \\
11 & 23.16 \\
12 & 17.65 \\
13 & 11.13 \\
14 & 7.12 \\
15 & 20.82 \\
16 & 14.96 \\
17 & 11.05 \\
\hline
\end{tabular}

CS is lower than the emergency introduced situation. This is because most of the nodes are avalanched resulting from cascading effect. Besides, the cascade propagation velocity $V$ is greater, which means more nodes are avalanched per time.

Secondly, Figure 6 describes the simulation results of the relative size $G$ with different recovery strategies. According to the relative size $G$, the RS 6 (the resources are distributed to the avalanched nodes according to the node pressure. If there are no avalanched nodes, the resources are distributed to the challenged nodes according to the node pressure.) can resist against cascading failures and protect the critical components in the network more effectively after the external resources are introduced into WDN. For example, when $\alpha=0.70, G_{6}=$ 0.7197 , the network connectivity is 0.1626 higher than that without the use of emergency resources. It can be found that RS 3 achieves poor performance. Although the challenged nodes may fail, it can still operate and supply water. Hence, resources should be firstly supplied for the avalanched nodes. The repair of avalanched nodes can effectively improve the entire robustness of WDN.

Thirdly, Figure 7 describes the simulation results of the avalanched size AS with different recovery strategies. The recovery strategies should be effective to reduce the avalanched nodes. Measures are taken to repair the network and enhance the robustness. It can be found in Figure 7 that there are situations in which the avalanched nodes with recovery strategies are higher than RS 7 (i.e., strategy without emergency resource), for example, when $\alpha=0.17 \sim$ 0.19 and $0.27 \sim 0.28$, AS (RS 1) > AS (RS 7); when $\alpha=0.17 \sim$ 0.29 , AS (RS 3) > AS (RS 7); and when $\alpha=0.17 \sim 0.20$, AS (RS 5) > AS (RS 7). RS 1 distributes resources to all nodes uniformly. It does not distinguish between the avalanched nodes and the challenged nodes. This leads to the allocation of resources lacking of focus. RS 3 distributes resources to the challenged nodes uniformly, which ignores the effective repair of the avalanched nodes. RS 5 distributes resources to the avalanched nodes according to node betweenness. Node betweenness considers the network topological structure but 


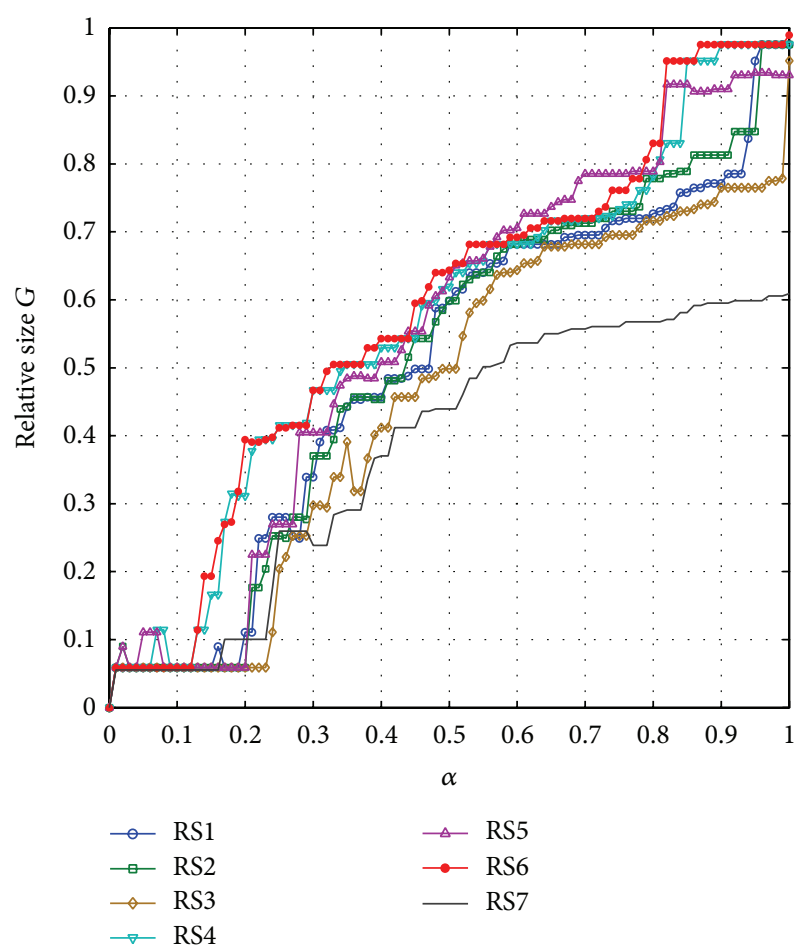

FIGURE 6: The simulation results of the relative size $G$ with different recovery strategies.

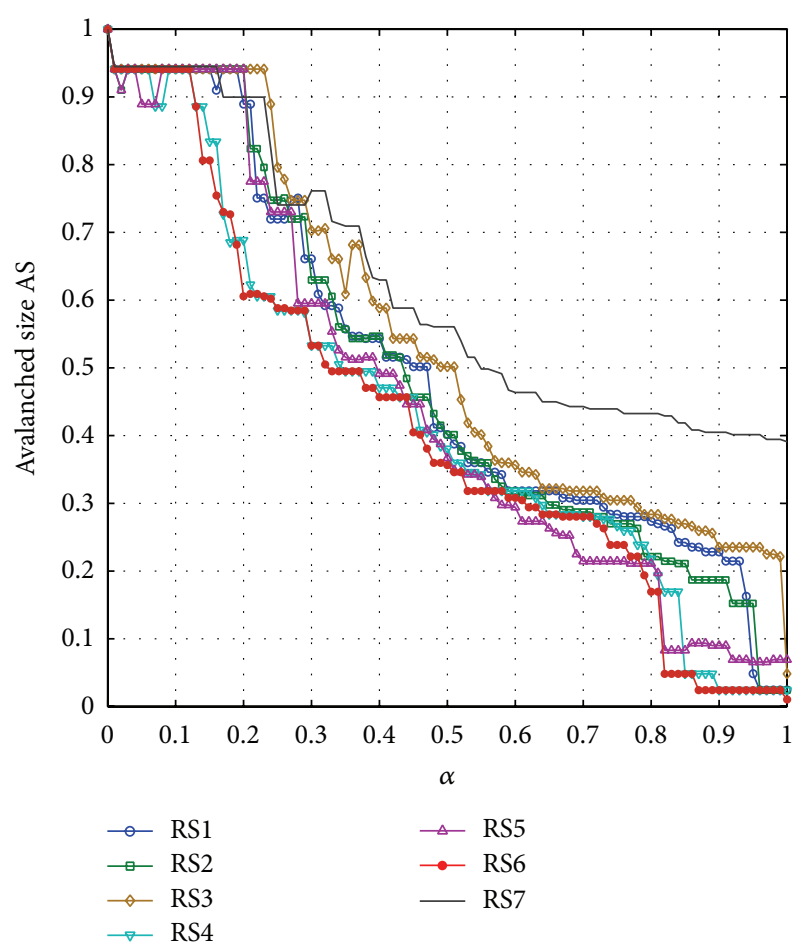

FIGURE 7: The simulation results of the avalanched size AS with different recovery strategies.

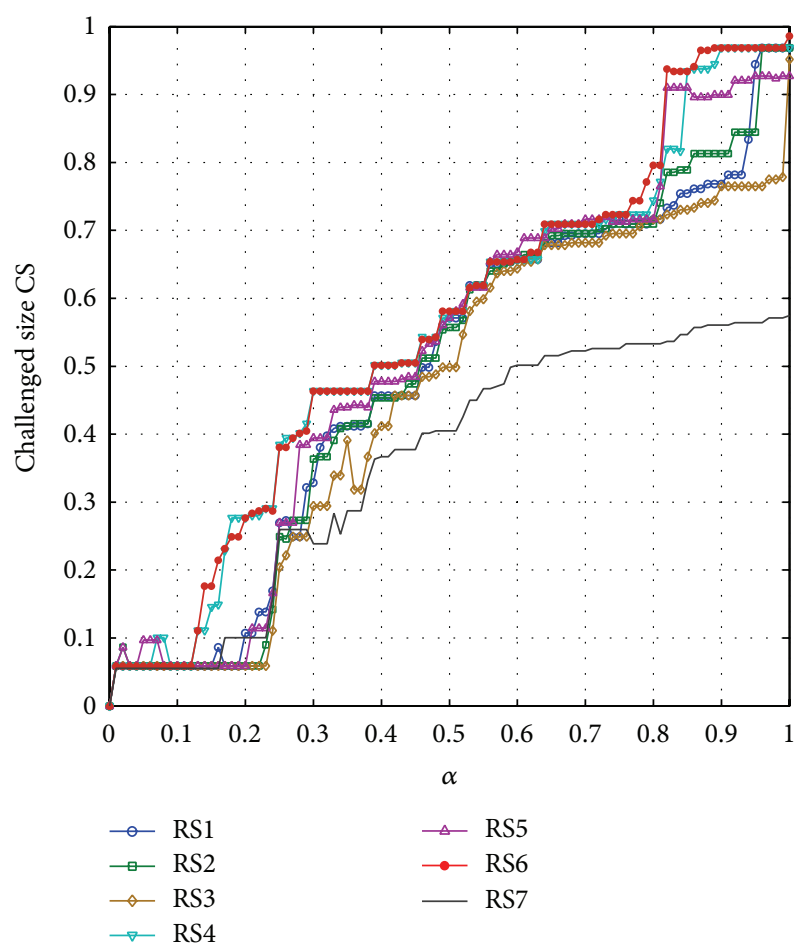

FIgURE 8: The simulation results of the challenged size CS with different recovery strategies.

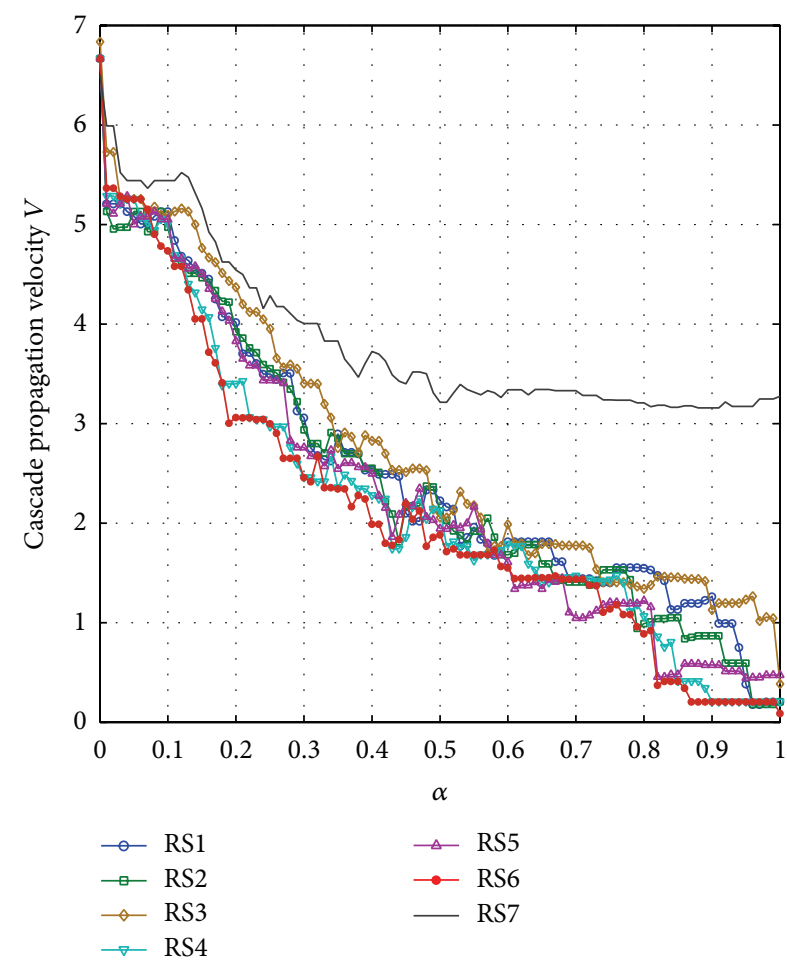

FIgURE 9: The simulation results of the cascade propagation velocity $V$ with different recovery strategies. 
TABLE 2: Different frequencies between RS 6 and other RSs.

\begin{tabular}{lccc}
\hline Frequency & $<0$ & $=0$ & $>0$ \\
\hline RS 6 - RS 1 & 0.8416 & 0.0297 & 0.1287 \\
RS 6 - RS 2 & 0.7921 & 0.0198 & 0.1881 \\
RS 6 - RS 3 & 0.9505 & 0.0297 & 0.0198 \\
RS 6 - RS 4 & 0.6337 & 0.1881 & 0.1782 \\
RS 6 - RS 5 & 0.8020 & 0.0099 & 0.1881 \\
RS 6 - RS 7 & 0.9901 & 0.0000 & 0.0099 \\
\hline
\end{tabular}

not the hydraulic property. Hence, there is a deviation on the distribution of the avalanched nodes. Moreover, the AS of RS 2 , RS 4, and RS 6 are lower than RS 7 in any state of $\alpha$. AS (RS 2) are higher than AS (RS 4) and AS (RS 6). Comparing RS 4 and RS 6, the times of AS (RS 6) < AS (RS 4) are 52; the times of AS (RS 6) = AS (RS 4) are 41; and the times of AS (RS 6) $>$ AS (RS 4) are 8 . Therefore, RS 6 can reduce the number of avalanched nodes more effectively and produce the best protection effect compared with other RSs.

Fourthly, Figure 8 describes the simulation results of the challenged size CS with different recovery strategies. When $\alpha$ ranges from 0.53 to 0.8 , the challenged nodes arising from the six RSs are identical. When $\alpha$ is smaller than 0.53 , RSs 4 and 6 produce more challenged nodes compared with the other RSs. RS 3 is capable of lessening the challenged nodes, mainly because this strategy has allocated resources to the challenged ones. However, on the contrary, RS 3 shows poor performance in terms of the relative and the avalanched size.

Fifthly, Figure 9 describes the simulation results of the cascade propagation velocity $V$ with different recovery strategies. The cascade propagation velocity $V$ measures the quantity of avalanched nodes in unit time. $V$ considers the total avalanched nodes after cascade-effect stops and the cascading spread time. Therefore, the smaller the $V$ is, the less the avalanched nodes per time produced. To better compare the recovery strategies, the difference frequencies between RS 6 and other RSs are calculated. Table 2 shows the result. Columns 2, 3, and 4 in Table 2 describe the frequencies indicating that $V$ (RS 6) is smaller than the other RSs, $V$ (RS 6) is equal to the other RSs, and $V$ (RS 6) is larger than the other RSs, respectively. From data in Table 2, RS 6 has the lowest number of avalanched nodes per time, which has effectively decreased the avalanched velocity of the cascading failures.

As shown in Figures 6-9, uniform recovery distribution according to challenged nodes (i.e., RS 3) has the lowest ability to resist against cascading failures. In this regard, however, the uniform recovery distribution of all nodes (i.e., RS 1) is parallel to the recovery distribution of all nodes according to node betweenness (i.e., RS 2). RS 4 and RS 6 produce good result. According to the analysis discussed above, the RS 6 can heighten the connectivity of the system and reduce the avalanched nodes and the number of the avalanched nodes that emerge in unit time effectively. Though RS 6 cannot lessen the challenged nodes, the challenged nodes can still supply water to guarantee the functions of WDN. Therefore, the RS 6 is capable of resisting against the cascading failures of WDN effectively.

\section{Conclusion}

WDN constitutes a significant part in infrastructure system. In this paper, attention is paid to studying the recovery strategies of urban WDN with cascading failures. The choice of the best strategy to fight the cascading failures is discussed. The case study manifests that the recovery strategy of node pressure, that is, the RS 6, is able to improve the connectivity of the WDN. Besides, this strategy can reduce the avalanched nodes and the number of avalanched nodes per time. The result shows that the recovery strategy based on node pressure is superior to that based on node betweenness. WDN is a kind of physical network, which is different from virtual network. Focus should be paid not only on the network topological, but also on the hydraulic property. The node pressure strategy considers both the topology structure and the balance of water supply and demand, which makes this strategy superior to others.

Further research should focus on the resource generation function to study the influence of time-based recovery strategy. To resist against the propagation of the cascading failures in WDN, efforts should be spent in establishing resource and cost constraint functions to study how to restore the network components effectively by limited resources and limited cost. In addition, to recognize node conditions, monitoring device with the functions of on-line monitoring and sensors for detecting pressure and flow should be installed on the real WDN. With the help of monitoring, the cascade-based emergency model can provide a more realistic result.

\section{Conflict of Interests}

The authors declare that there is no conflict of interests regarding the publication of this paper.

\section{Acknowledgments}

This work was supported by the National Natural Science Foundation of China (no. 51208081) and the Fundamental Research Founds for Beijing Jiaotong University (no. B15RC00010). The authors also gratefully acknowledge the helpful comments and suggestions of the reviewers, which have improved the presentation.

\section{References}

[1] R. Pastor-Satorras, A. Vázquez, and A. Vespignani, "Dynamical and correlation properties of the internet," Physical Review Letters, vol. 87, no. 25, Article ID 258701, 4 pages, 2001.

[2] J.-W. Wang and L.-L. Rong, "Robustness of the western United States power grid under edge attack strategies due to cascading failures," Safety Science, vol. 49, no. 6, pp. 807-812, 2011.

[3] Y. Zhang and N. Yang, "Research on robustness of R\&D network under cascading propagation of risk with gray attack information," Reliability Engineering and System Safety, vol. 117, no. 9, pp. 1-8, 2013.

[4] S. L. Yang, N. S. Hsu, P. W. F. Louie, and W. W. G. Yeh, "Water distribution network reliability: connectivity analysis," Journal of Infrastructure Systems, vol. 2, no. 2, pp. 54-64, 1996. 
[5] P. Li, B.-H. Wang, H. Sun, P. Gao, and T. Zhou, "A limited resource model of fault-tolerant capability against cascading failure of complex network," European Physical Journal B, vol. 62, no. 1, pp. 101-104, 2008.

[6] A. E. Motter and Y. C. Lai, "Cascade-based attacks on complex networks," Physical Review E-Statistical, Nonlinear, and Soft Matter Physics, vol. 66, no. 6, Article ID 065102, 4 pages, 2002.

[7] I. Hernandez-Fajardo and L. Dueñas-Osorio, "Probabilistic study of cascading failures in complex interdependent lifeline systems," Reliability Engineering \& System Safety, vol. 111, no. 2, pp. 260-272, 2013.

[8] T. Adachi, Impact of cascading failures on performance assessment of civil infrastructure systems [Ph.D. thesis], Georgia Institute of Technology, Atlanta, Ga, USA, 2007.

[9] T. Adachi and B. R. Ellingwood, "Serviceability of earthquakedamaged water systems: effects of electrical power availability and power backup systems on system vulnerability," Reliability Engineering \& System Safety, vol. 93, no. 1, pp. 78-88, 2008.

[10] R. Sitzenfrei, M. Mair, M. Möderl, and W. Rauch, "Cascade vulnerability for risk analysis of water infrastructure," Water Science and Technology, vol. 64, no. 9, pp. 1885-1891, 2011.

[11] A. Yazdani and P. Jeffrey, "Complex network analysis of water distribution systems," Chaos, vol. 21, no. 1, Article ID 016111, 11 pages, 2011.

[12] A. Yazdani, R. A. Otoo, and P. Jeffrey, "Resilience enhancing expansion strategies for water distribution systems: a network theory approach," Environmental Modelling \& Software, vol. 26, no. 12, pp. 1574-1582, 2011.

[13] K. A. Hawick, "Water distribution network robustness and fragmentation using graph metrics," in Proceedings of the International Conference on Water Resource Management (AfricaWRM '12), pp. 304-310, September 2012.

[14] Q. Shuang, M. Zhang, and Y. Yuan, "Node vulnerability of water distribution networks under cascading failures," Reliability Engineering and System Safety, vol. 124, no. 1, pp. 132-141, 2014.

[15] Q. Shuang, M. Zhang, Y. Yuan, and V. Magar, "Performance and reliability analysis of water distribution systems under cascading failures and the identification of crucial pipes," PLOS ONE, vol. 9, no. 2, p. 11, 2014.

[16] J. A. Bondy and U. S. R. Murty, Graph Theory with Applications, Elsevier, New York, NY, USA, 1976.

[17] J. M. Wagner, U. Shamir, and D. H. Marks, "Water distribution reliability-simulation methods," Journal of Water Resources Planning and Management, vol. 114, no. 3, pp. 276-294, 1988.

[18] A. Ostfeld, D. Kogan, and U. Shamir, "Reliability simulation of water distribution systems-single and multiquality," Urban Water, vol. 4, no. 1, pp. 53-61, 2002.

[19] B. Zhuang, K. Lansey, and D. Kang, "Resilience/availability analysis of municipal water distribution system incorporating adaptive pump operation," Journal of Hydraulic Engineering, vol. 139, no. 5, pp. 527-537, 2013.

[20] D. Laucelli, L. Berardi, and O. Giustolisi, "Assessing climate change and asset deterioration impacts on water distribution networks: demand-driven or pressure-driven network modeling?" Environmental Modelling \& Software, vol. 37, pp. 206-216, 2012.

[21] L. A. Rossman, EPANET 2 User's Manual, U.S. Environmental Protection Agency, Cincinnati, Ohio, USA, 2000.

[22] E. Todini and S. Pilati, "A gradient method for the analysis of pipe networks," in Proceedings of the International Conference on Computer Applications for Water Supply and Distribution, Leicester Polytechnic, UK, 1987.

[23] O. Giustolisi and T. M. Walski, "Demand components in water distribution network analysis," Journal of Water Resources Planning and Management, vol. 138, no. 4, pp. 356-367, 2012.

[24] O. Giustolisi and D. Laucelli, "Water distribution network pressure-driven analysis using the enhanced global gradient algorithm (EGGA)," Journal of Water Resources Planning and Management, vol. 137, no. 6, pp. 498-510, 2011.

[25] R. Albert, H. Jeong, and A.-L. Barabási, "Error and attack tolerance of complex networks," Nature, vol. 406, no. 6794, pp. 378-382, 2000.

[26] P. Crucitti, V. Latora, and M. Marchiori, "Model for cascading failures in complex networks," Physical Review E: Statistical, Nonlinear, and Soft Matter Physics, vol. 69, no. 4, Article ID 045104, 2004.

[27] S. D. Li, Research on the Mechanism of Cascading Dynamics and Behaviors of Complex Networks, Beijing University of Posts and Telecommunications, Beijing, China, 2012.

[28] C.-L. Chai, X. Liu, W. J. Zhang, and Z. Baber, "Application of social network theory to prioritizing Oil \& Gas industries protection in a networked critical infrastructure system," Journal of Loss Prevention in the Process Industries, vol. 24, no. 5, pp. 688694, 2011.

[29] L. Dueñas-Osorio and S. M. Vemuru, "Cascading failures in complex infrastructure systems," Structural Safety, vol. 31, no. 2, pp. 157-167, 2009.

[30] J.-W. Wang and L.-L. Rong, "Cascade-based attack vulnerability on the US power grid," Safety Science, vol. 47, no. 10, pp. 1332$1336,2009$.

[31] S. Li, L. Li, Y. Yang, and Q. Luo, "Revealing the process of edgebased-attack cascading failures," Nonlinear Dynamics, vol. 69, no. 3, pp. 837-845, 2012.

[32] M. Ouyang, M.-H. Yu, X.-Z. Huang, and E.-J. Luan, "Emergency response to disaster-struck scale-free network with redundant systems," Physica A: Statistical Mechanics and Its Applications, vol. 387, no. 18, pp. 4683-4691, 2008. 


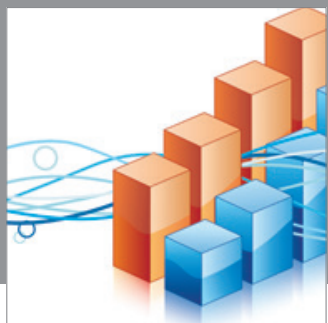

Advances in

Operations Research

mansans

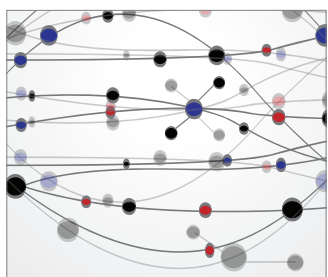

The Scientific World Journal
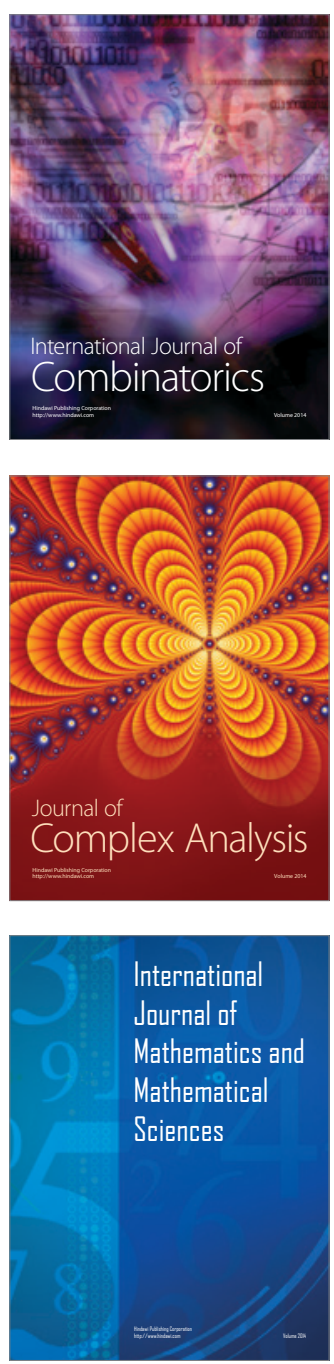
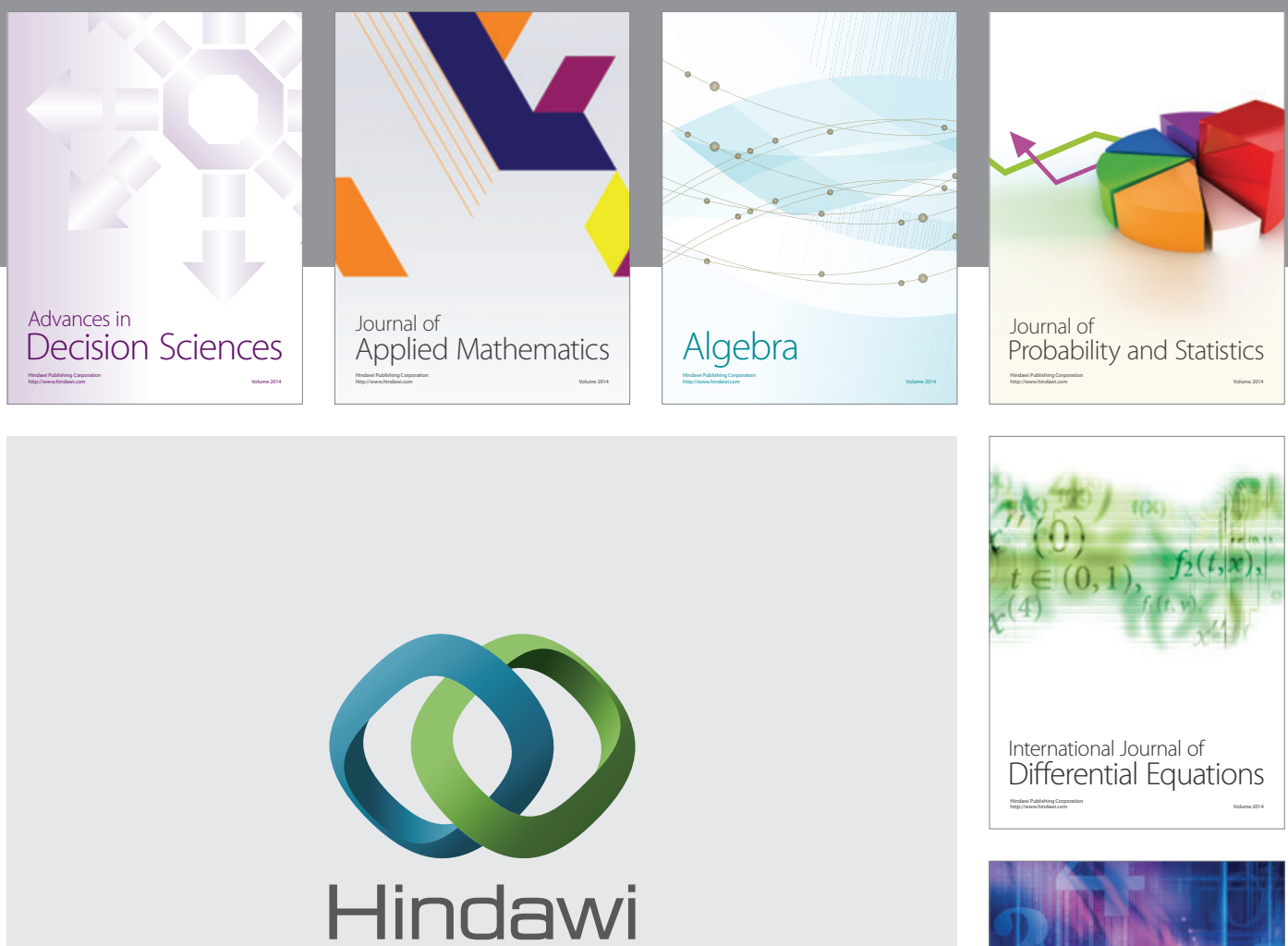

Submit your manuscripts at http://www.hindawi.com
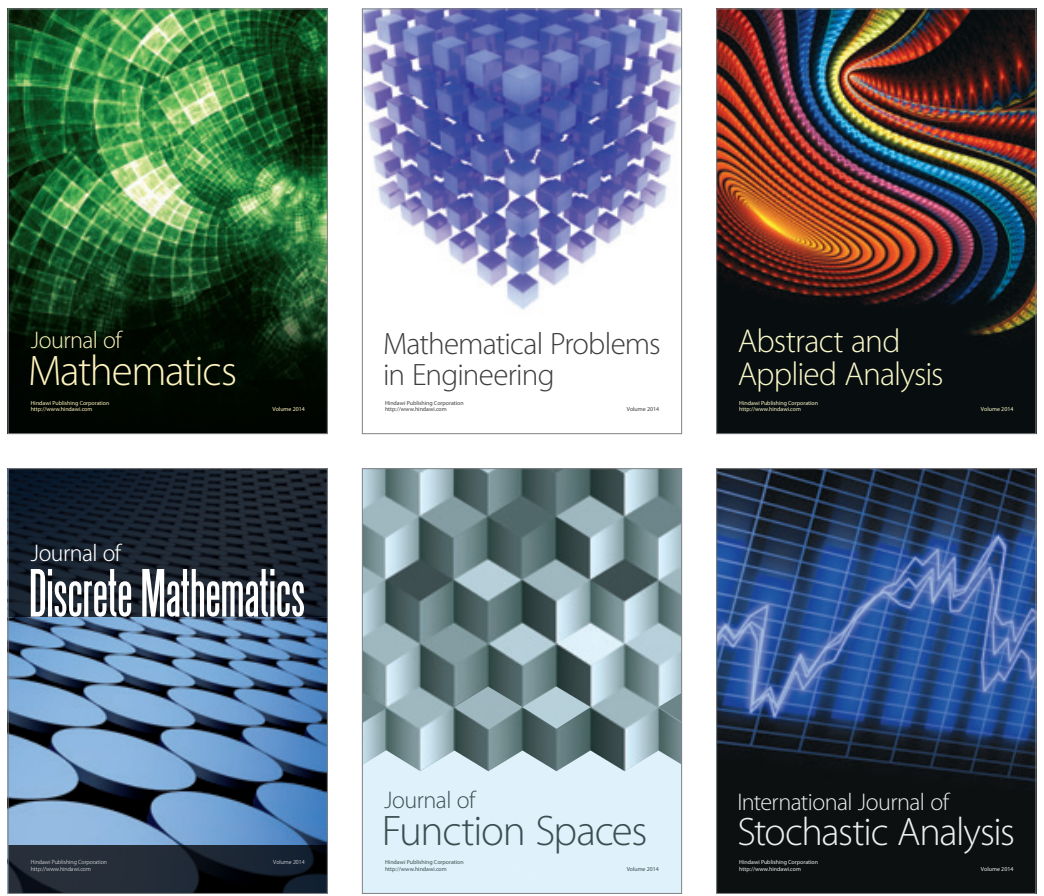

Journal of

Function Spaces

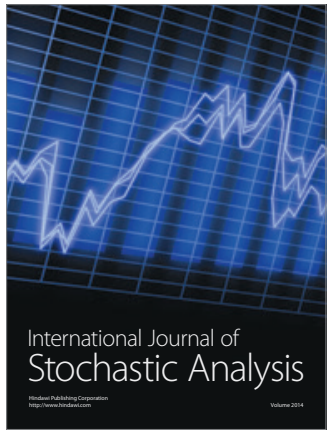

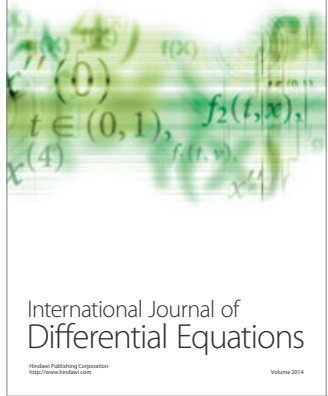
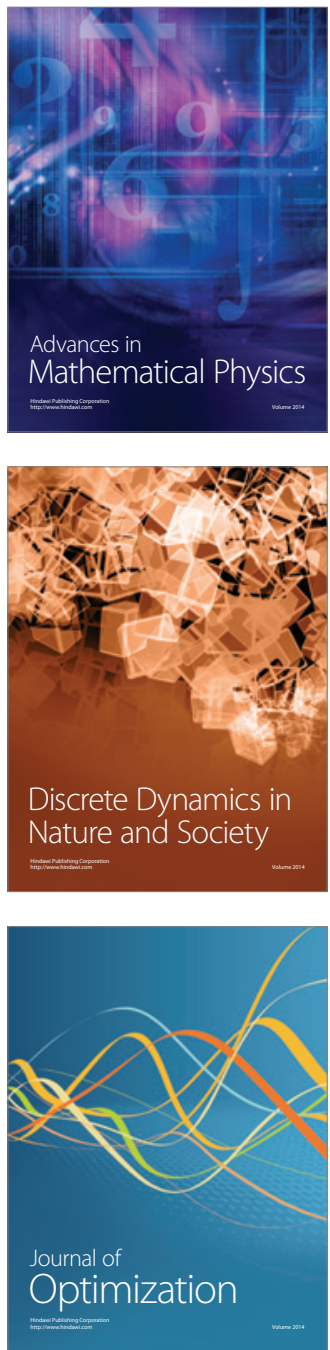